\title{
ABUNDANCES AND NEBULAR AND CENTRAL STAR MASSES FOR MAGELLANIC CLOUD PLANETARY NEBULAE.
}

\author{
N.A. WALTON, M.J. BARLOW, \\ \& D.J. MONK \\ Department of Physics \& Astronomy \\ University College London \\ Gower Street, London WC1E 6BT
}

\author{
R.E.S. CLEGG \\ Royal Greenwich Observatory \\ Madingley Road \\ Cambridge CB3 OEZ
}

\begin{abstract}
We present the results of a spectroscopic study of planetary nebulae (PN) in the Magellanic Clouds. The optical survey of $\mathrm{He}, \mathrm{N}, \mathrm{O}$, and Ne abundances by Monk et al. (1988) has been updated by higher S/N AAT optical data. In addition, carbon and other elemental abundances have been derived from the $I U E$ spectra of $38 \mathrm{PN}$. Ionized nebular masses have been derived for $80 \mathrm{PN}$. The ionised mass versus nebular electron density plot shows that planetary nebulae become optically thin when their electron densities drop below $4500 \mathrm{~cm}^{-3}$. Below this density, the mean nebular hydrogen mass found for non-Type I PN is $0.22 \pm 0.08 \mathrm{M}_{\odot}$. Using Zanstra and energy-balance methods, the mean central star mass found for 14 SMC and LMC PN is $0.59 \pm 0.02 \mathrm{M}_{\odot}$.
\end{abstract}

$I U E$ and $A A T$ spectra of 38 planetary nebulae in the Magellanic Clouds have been analysed to derive ionic and total elemental abundances for a range of species. Our results are summarised in Tables 1 and 2 of Barlow (1990). The nitrogen abundances in the non-Type I nebulae are found to be consistent with the exposure of secondary nitrogen (produced by the $\mathrm{CN}$ cycle) by the first dredge-up, with $50 \%$ and $100 \%$ of the initial carbon having been converted to nitrogen in the LMC and SMC, respectively. All of the non-Type I PN have $\mathrm{C} / \mathrm{O}$ ratios significantly larger than unity, consistent with the exposure of primary carbon by the third dredge-up. The carbon enhancements are largest in the SMC, the galaxy with the lower metallicity.

The five LMC Type I PN (those with $\mathrm{N} / \mathrm{O}>0.5$ ) were found to have the same mean oxygen abundance as found for the non-Type I PN and $\mathrm{H}$ II regions in the LMC, implying that no large ON-cycle depletion of oxygen resulted from the second dredge-up. From a comparison of the $\mathrm{N}$ and $\mathrm{C}+\mathrm{N}$ abundances in the LMC Type I nebulae with those in LMC H II regions, it is concluded that the third dredge-up plus envelope-burning (which converted some of the dredged-up carbon to nitrogen) occurred in the Type I progenitor stars. Fig. 1 shows that two of the five LMC Type I nebulae have $\mathrm{C} / \mathrm{O}>1$. The Ne/O ratio is found to be enhanced in LMC Type I PN by $0.19 \pm 0.03$ dex relative to LMC non-Type I $\mathrm{PN}$ and $\mathrm{H}$ II regions.

Ionized nebular hydrogen masses have been derived for 80 Magellanic Cloud PN using dereddened $\mathrm{H} \beta$ fluxes and accurate [O II] $3726,29 \AA$ doublet ratio electron densities. A plot of ionized hydrogen mass versus nebular electron density indicates that the expanding PN become optically thin when their electron densities drop below $4500 \mathrm{~cm}^{-3}$. Table 1 summarises the mean hydrogen masses found for SMC and LMC PN over a number of density ranges. The mean nebular hydrogen mass found for non-Type I PN is the same in the SMC and LMC and equal to $0.217 \pm 0.077 \mathrm{M}_{\odot}$. 


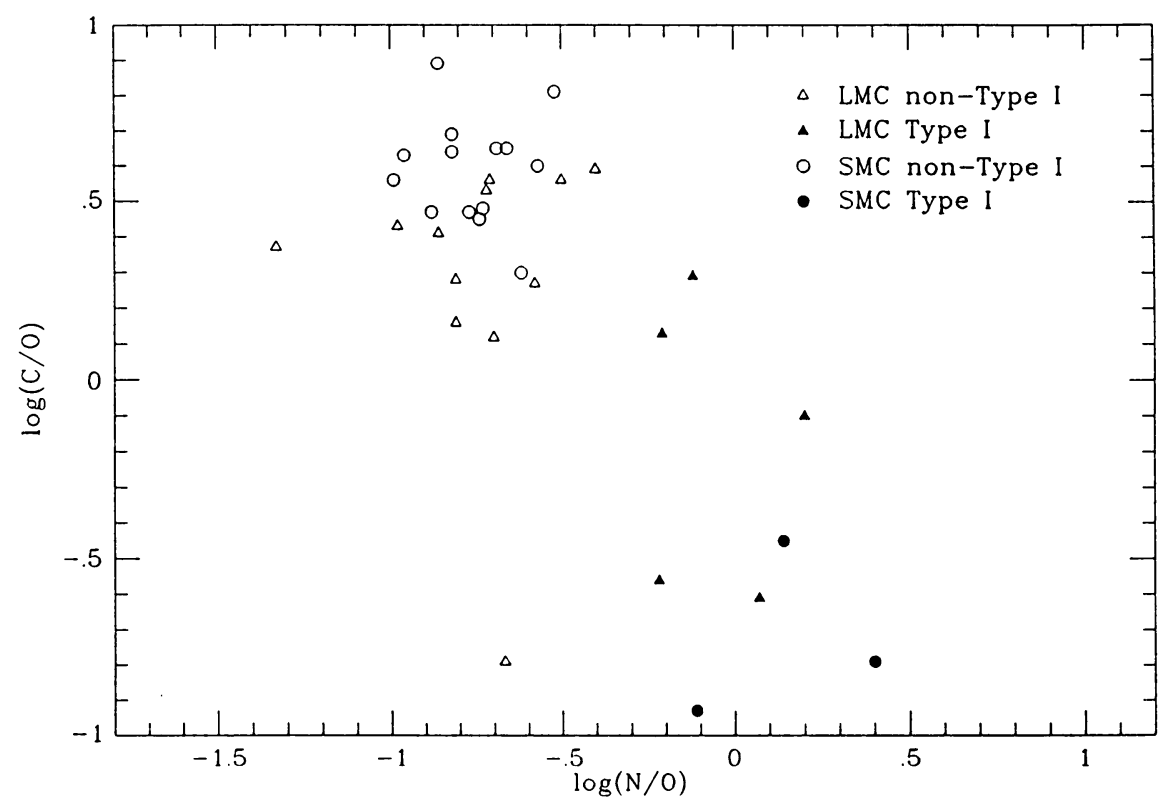

Figure 1: The relationship between $\log (\mathrm{C} / \mathrm{O})$ and $\log (\mathrm{N} / \mathrm{O})$ for the sample of Magellanic Cloud planetary nebulae.

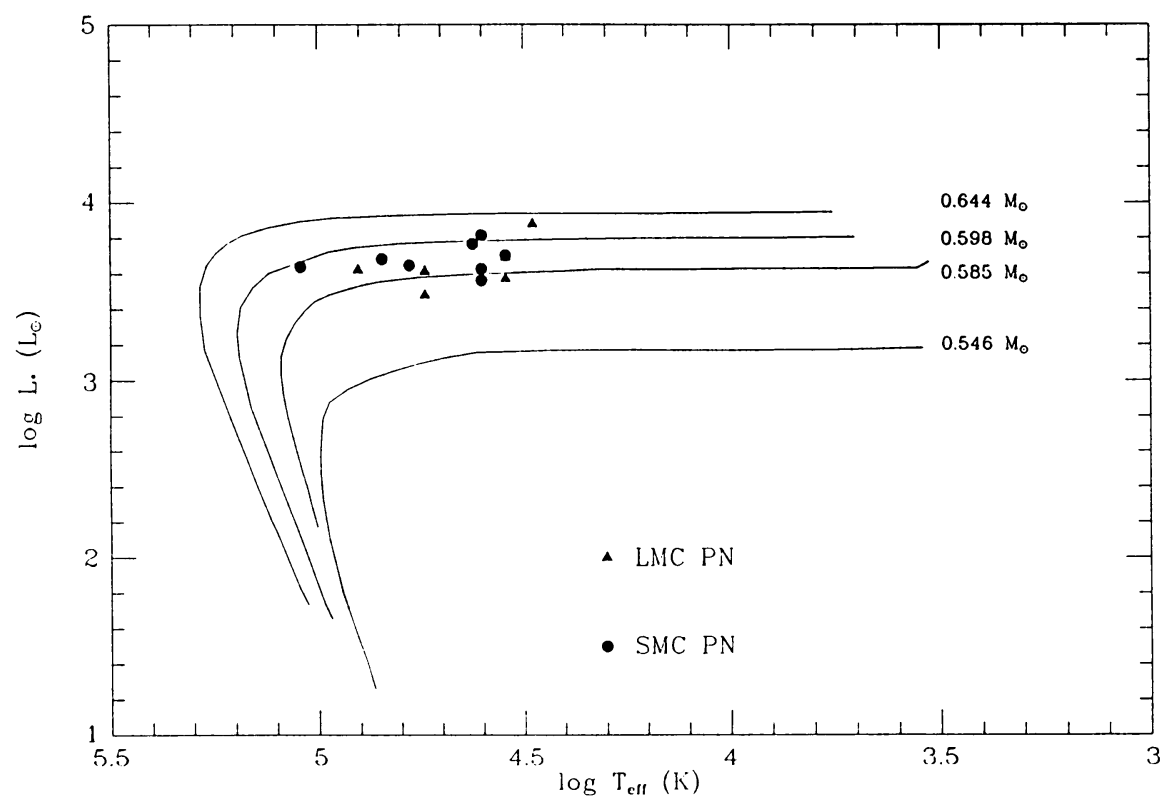

Figure 2: The central stars of a number of the Magellanic Cloud planetary nebulae are shown plotted on an H-R diagram. Schoenberner evolutionary tracks for central stars having a range of core masses are also plotted. 


\section{Table 1: Ionized Hydrogen Masses}

\begin{tabular}{llcc} 
& PN Sample & $\begin{array}{c}\text { Electron Density } \\
\left(\mathrm{cm}^{-3}\right)\end{array}$ & $\begin{array}{c}\text { Mean Ionized Hydrogen Mass } \\
\left(\mathrm{M}_{\odot}\right)\end{array}$ \\
& & & \\
7 & SMC non-Type I & $<2500$ & $0.214 \pm 0.099$ \\
7 & SMC non-Type I & $2500<\mathrm{n}_{\mathrm{e}}<4500$ & $0.220 \pm 0.045$ \\
15 & SMC non-Type I & $<4500$ & $0.217 \pm 0.079$ \\
& & & \\
9 & LMC non-Type I & $<2500$ & $0.217 \pm 0.084$ \\
11 & LMC non-Type I & $2500<\mathrm{n}_{\mathrm{e}}<4500$ & $0.217 \pm 0.067$ \\
20 & LMC non-Type I & $<4500$ & $0.217 \pm 0.075$ \\
35 & Total non-Type I & $<4500$ & $0.217 \pm 0.077$ \\
& & & \\
3 & SMC Type I & $<4500$ & $0.280 \pm 0.062$ \\
7 & LMC Type I & $<4500$ & $0.359 \pm 0.093$ \\
\multicolumn{3}{c}{ The adopted distance moduli are: LMC $18^{\mathrm{m}} \cdot 35$ and SMC $18^{\mathrm{m}} \cdot 8$}
\end{tabular}

From optical and ultraviolet spectra, we have derived central star effective temperatures, surface gravities and masses using the Zanstra and energy-balance methods. Fig. 2 shows our results for 14 central stars, plotted on an H-R diagram along with a number of theoretical evolutionary tracks for a range of core masses. The central star mass distribution is found to be very narrow. The mean mass found for all 14 central stars is $0.585 \pm 0.016$ $\mathrm{M}_{\odot}$; the eight SMC central stars give $0.587 \pm 0.013 \mathrm{M}_{\odot}$ and the six LMC central stars give $0.583 \pm 0.020 \mathrm{M}_{\odot}$.

\section{References}

Barlow, M. J., 1990. These Proceedings.

Monk, D. J., Barlow, M. J. \& Clegg, R. E. S., 1988. Mon. not. R. astr. Soc., 234, 583. 